\title{
Quality-control method for the determination of biological activity of engineered calcineurin subunit $B$
}

\author{
Xinchang Shi ${ }^{1 \dagger}$, Huan Yang ${ }^{2 \dagger}, \mathrm{Li} \mathrm{Xu}^{1}$, Xiang $\mathrm{Li}^{2}$, Zongwen Huang ${ }^{3}$, Yudong $\mathrm{Han}^{3}$, Qun Wei ${ }^{2 *}$ \\ \& Chunming Rao ${ }^{1:}$ \\ ${ }^{1}$ Key Laboratory of the Ministry of Health for Research on Quality and Standardization of Biotech Products, National Institutes for Food and \\ Drug Control, Beijing 100050, China; \\ ${ }^{2}$ Department of Biochemistry and Molecular Biology, Beijing Normal University, Gene Engineering and Biotechnology Beijing Key Laboratory, \\ Beijing 100875, China; \\ ${ }^{3}$ Haikou Qili Pharmaceutical CO., LTD., Haikou 570216, China
}

Received December 21, 2015; accepted February 1, 2016; published online April 1, 2016

\begin{abstract}
The aim of this study was to establish a quality-control method for calcineurin subunit B (CNB) biological activity determinations. CNB enhances the $p$-nitrophenylphosphate (pNPP) dephosphorylating activity of calcineurin subunit A $\Delta 316$ mutant $(\mathrm{CNA} \triangle 316)$. A series of CNB concentrations were fitted to a four-parameter equation to calculate the corresponding pNPP maximum dephosphorylation rates. Values were calculated based on biological activity references using a parallel line method. The method was then validated for accuracy, precision, linearity, linear range, sensitivity, specificity, and robustness. The recovery results were greater than $98 \%$. Intra-plate precision was $6.7 \%$, with inter-plate precision of $10.8 \%$. The coefficient of determination was greater than 0.98 . The linear range was $0.05-50 \mu \mathrm{g} \mathrm{mL}^{-1}$, with sensitivity of $50 \mu \mathrm{g} \mathrm{mL} \mathrm{m}^{-1}$. Tested cytokines did not induce CNA $\triangle 316$ dephosphorylation of pNPP. The chosen CNA $\Delta 316$ concentration range did not affect activity determinations.
\end{abstract}

calcineurin subunit B, biological activity, method establishment, method validation, quality control

Citation: Shi, X., Yang, H., Xu, L., Li, X., Huang, Z., Han, Y., Wei, Q., and Rao, C. (2016). Quality-control method for the determination of biological activity of engineered calcineurin subunit B. Sci China Life Sci 59, 584-588. doi: 10.1007/s11427-016-5037-2

\section{INTRODUCTION}

$\mathrm{CNB}$ is a regulatory subunit of calcineurin $(\mathrm{CN})$ with a well-established function of regulating the phosphatase catalytic activity of CN (Rusnak and Mertz, 2000; Aramburu et al., 2000). CNB also plays additional, non-regulatory roles. For example, CNB can significantly extend the survival of H22 hepatoma ascites- or S180 sarcoma tumor-bearing mice and exerts a well-defined therapeutic effect on the liver and the lung in nude mouse cancer model (Wei et al., 2002). CNB can also stimulate the im-

$\dagger$ Contributed equally to this work

*Corresponding author (email: weiq@bnu.edu.cn)

†Corresponding author (email: raocm@nicpbp.org.cn) mune system, as it increases the natural killer activity of murine spleen lymphocytes, promotes peritoneal macrophage proliferation, enhances phagocytic activity, and increases the secretion of cytokines (Wei et al., 2002; Jin et al., 2005). Further studies revealed that CNB interacts with integrin $\alpha \mathrm{M}$ and increases TNF-related apoptosis-inducing ligand (TRAIL) expression in macrophages, thereby inducing tumor cell apoptosis (Liu et al., 2012; Su et al., 2012). Furthermore, CNB promotes TNFinduced apoptosis, possibly by affecting mitochondrial function (Cheng et al., 2012). The remarkable tumor suppressive effect and the extremely low incidence of side effects of CNB administration render it a promising anti-cancer drug. CNB has been developed as a genetically 
engineered drug, patented in China and the US (Wei et al., 2003; Wei et al., 2006). A preparatory CNB production process has been initiated, and the methods for evaluation of CNB molecular weight, isoelectric point, $\mathrm{N}$-terminal amino acid sequence, purity, and other objective indicators of quality control have been standardized. However, no detection method for $\mathrm{CNB}$ activity has been established. In the context of patient treatment, the establishment of a specific CNB activity determination method is particularly important. According to previous studies, CNB regulates the activity of $\mathrm{CN}$ by interacting with CNA, the catalytic subunit of CN (Rusnak and Mertz, 2000; Aramburu et al., 2000). CNB binds to the BBH domain of CNA to regulate its activity and stability (Reiter et al., 1999). Binding of calcium ions to the four calcium-binding sites of CNB triggers a conformation change in CNA, activating CN (Yang et al., 2000; Feng et al., 1998). Therefore, CNA conformational change may be a good choice as CNB activity determination target.

Here, we establish a practical CNB activity determination method. CNA activity is low and this subunit is not suitable for industrial detection, storage, and transportation. We therefore used CNA $\Delta 316$ mutant, one of Loop $7 \mathrm{mu}-$ tants, which has a higher activity and stability than native CNA and retains the characteristics of CNA interaction with CNB (Wei et al., 1997; Maynes et al., 2004). The method was developed based on the observation that CNB promotes the ability of CNA $\Delta 316$ to dephosphorylate $p$-nitrophenylphosphate (pNPP) (Wei et al., 1997). The method is easy to implement, with high stability and quantitative accuracy, laying a solid foundation for CNB drug development.

\section{RESULTS}

\section{An assay for measuring the activity of CNB}

The relationship between the absorption values in each well and reaction time was linear, and comprised a typical enzyme kinetics curve. Initially, the slope of the plotted curve was slightly slow and unstable. When the reaction temperature equilibrated at the set temperature, the curve reached its maximum slope. With a sufficiently long reaction time, the curve leveled off due to decreasing substrate concentrations.

The reaction rate correlated with the amount of enzyme when the amount of substrate was not limiting. Thus, $V_{\max }$ was proportional to the amount of the enzyme. The results are shown in Figure 1. In the assay, total enzyme activity comprised combined enzyme activity of CNA $\Delta 316$ subunit and the CNA $\triangle 316-\mathrm{CNB}$ complex.

The relationship between the concentration of $\mathrm{CNB}$ and $V_{\max }$ was linear, as determined by the four-parameter regression equation. The lower asymptote reflects the amount of CNA $\triangle 316$ and the upper asymptote reflects the amount of CNA $\triangle 316-C N B$. The ratio of the maximum (upper asymptote) to minimum (the lower asymptote) values corresponds the proportion of CNA $\Delta 316$ activated by CNB (Figure 2).

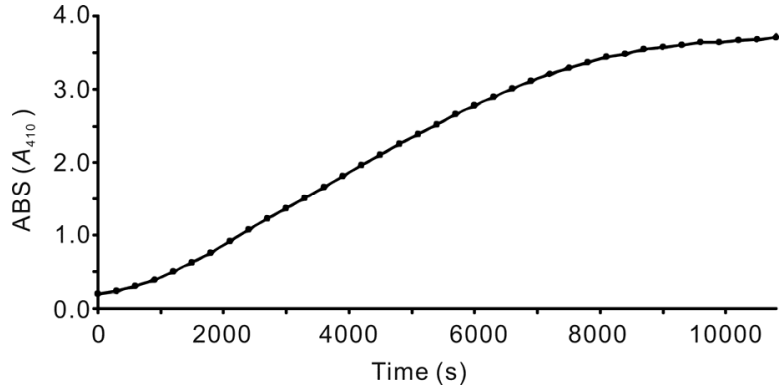

Figure 1 Enzyme kinetics curve relating reaction time to absorption measurements. Note: The kinetics curve represents an example of substrate decomposition by CNA316 promoted by CNB, read from one well of 96-well plate as absorbance versus time. Due to the changing temperature, the initial reaction rate is relatively slow. The middle of the curve approaches a straight line, where the reaction proceeds at the maximum rate $\left(V_{\max }\right)$. As the substrate is consumed, the reaction rate decreases.

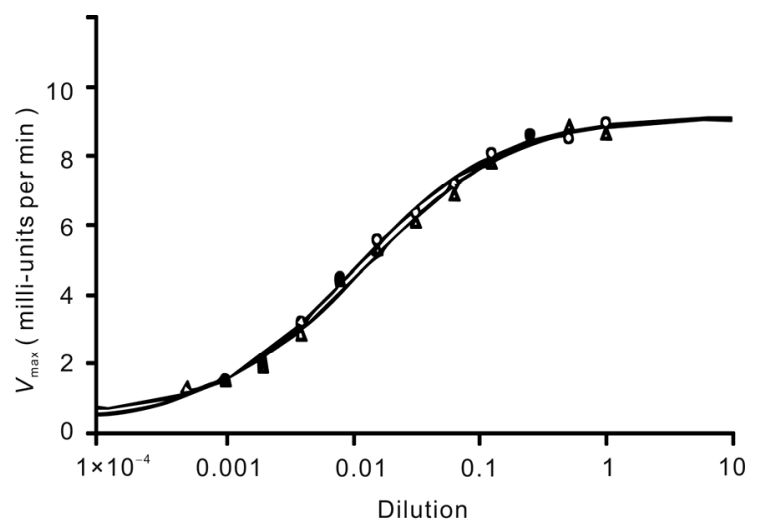

Figure 2 Dose-response curve between $\mathrm{CNB}$ dilution and $V_{\max }$. The $\mathrm{S}$-shaped curve reflects the maximum decomposition rate of pNPP at different concentrations (dilutions) of $\mathrm{CNB}$, where undiluted $\mathrm{CNB}$ preparation $=1 . \triangle \mathrm{CNA} 316$ and $\mathrm{pNPP}$ concentrations, and the temperature were constant. Two curves in the figure were used for biological activity value referral: $\bigcirc$, represents data for $\mathrm{CNB}$ reference material. $\Delta$, represents data for CNB sample.

The data revealed that the activity of CNA could be enhanced by up to 18.6 fold in the presence of CNB. The two four-parameter regression curves can be used to refer the activity values of $\mathrm{CNB}$ samples to $\mathrm{CNB}$ standard, and for quality control of CNB.

\section{Parallel transformation analysis}

Dose-effect lines of the reference material (standards) and CNB samples, plotted as $V_{\max }$ vs. logarithmic-transformed protein dilutions, are shown in Figure 3. The value was 0.3709 . The corresponding $P$ value was 0.7146 , greater than 0.05 . Therefore, the difference between the reference material and the CNB sample was not significant. The two lines were parallel to each other, and the reactions were consistent with each other.

\section{Accuracy assessments}

The recovery rate reached $98 \%$, close to $100 \%$, indicating a minimal effect of the solution formulation of CNB standard 


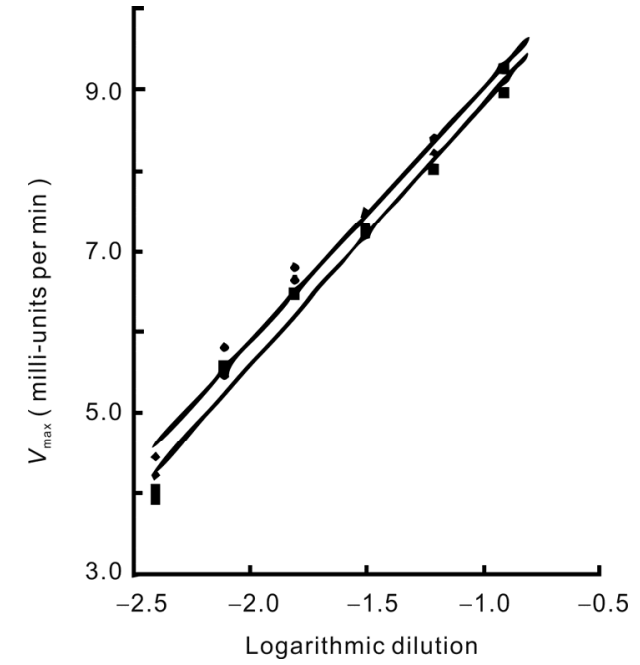

Figure 3 Analysis of reaction consistency. The maximum reaction rate obtained from the middle segment of the S-shaped curve in Figure 2 shows a linear relationship with logarithmic-transformed dilutions of CNB. The curves were used to analyze parallelism and to assess consistency of CNB samples and CNB reference material. $\diamond$, represent data for CNB reference material. Line equation for data fitting, $Y=3.138 X+12.129\left(R^{2}=0.986\right)$. $\square$, represent data for $\mathrm{CNB}$ sample. Line equation for data fitting, $Y=3.204 X+12.017\left(R^{2}=0.982\right)$.

and CNB test sample on the test system. This indicated that the method is highly accurate.

\section{Precision assessments}

Results from several independent experiments were analyzed statistically. The intra-plate precision was less than $6.7 \%$, and the inter-plate precision was less than $10.8 \%$. These results indicated that the precision of the method was adequate.

\section{Linearity testing}

After fitting data from several experiments to the four-parameter regression equation, the coefficient of determination $\left(R^{2}\right)$ was greater than 0.98 .

\section{Sensitivity assessment}

The lowest CNB concentration beyond the upper inflection-point of the four-parameter regression curve was approximately $50 \mu \mathrm{g} \mathrm{mL}^{-1}$. Thus, the sensitivity of this method was $50 \mu \mathrm{g} \mathrm{mL}^{-1}$. Beyond that point, most CNA $\Delta 316$ was bound to $\mathrm{CNB}$. The nominal concentration $\left(\mu \mathrm{g} \mathrm{mL} \mathrm{m}^{-1}\right)$ ratio of CNB to CNA $\triangle 316$ was approximately $10: 1$, while the molar concentration ratio of $\mathrm{CNB}$ to $\mathrm{CNA} \triangle 316$ was approximately $32: 1$.

\section{Specificity assessment}

Cytokines, such as rhG-CSF, rhIFN- $\alpha 2 b$, and rhIL-2, did not activate the enzymatic activity of CNA $\Delta 316$ (data not shown). The ability of CNB to activate CNA $\Delta 316$ was effectively inhibited by polyclonal anti-CNB antibodies (data not shown).

\section{Robustness testing}

This method was designed to refer the activity values of CNB samples to the CNB standard. Due to the reference material applied, the experimental temperature, reaction time, and substrate concentrations were robust. However, the experiment was affected by the amount of $\mathrm{CNA} \Delta 316$. We tested different CNA $\triangle 316$ concentrations in the system. This revealed that CNA $\triangle 316$ concentrations between 2.5 and $10 \mu \mathrm{g} \mathrm{mL}^{-1}$ did not affect the shape of the curve. The effect of various CNA $\Delta 316$ concentrations is shown in Figure 4.

\section{DISCUSSION}

The chromogenic substrate, pNPP, is dephosphorylated (decomposed) by CNA to produce a yellow substance with an absorption peak at $405 \mathrm{~nm}$ (Chaudhuri et al., 2013; Kissmehl et al., 1997). CNA $\Delta 316$ is a mutated form of CNA that retains the activity of the native subunit and its ability to be activated by subunit B (Wei et al., 1997; Maynes et al., 2004). The mutation increases the stability of CNA subunit, making it more suitable for quality control experiments.

This study was designed to establish a quality control assay for measuring the activity of the engineered CNB product. In other words, to characterize CNB samples using CNB reference materials. The formula and calculation of biological activity value referrals corresponded to the method used for assays with recombinant cytokines, described in the general of Chinese Pharmacopoeia. In the general, enzyme dilution or dilution factor were used as the horizontal ordinate of a four-parameter curve (National Pharmacopoeia Committee, 2015). We determined the activity of CNB samples using the following formula: $P \mathrm{~s}=\operatorname{Pr} \times((D \mathrm{~s} \times E \mathrm{~s}) /(D \mathrm{r} \times E \mathrm{r}))$. In this formula, $P \mathrm{~s}$ and $P r$ represent the biological activities of a CNB sample and CNB reference material (U per $\mathrm{mL}$ ), respectively; $D$ s and $D$ r rep-

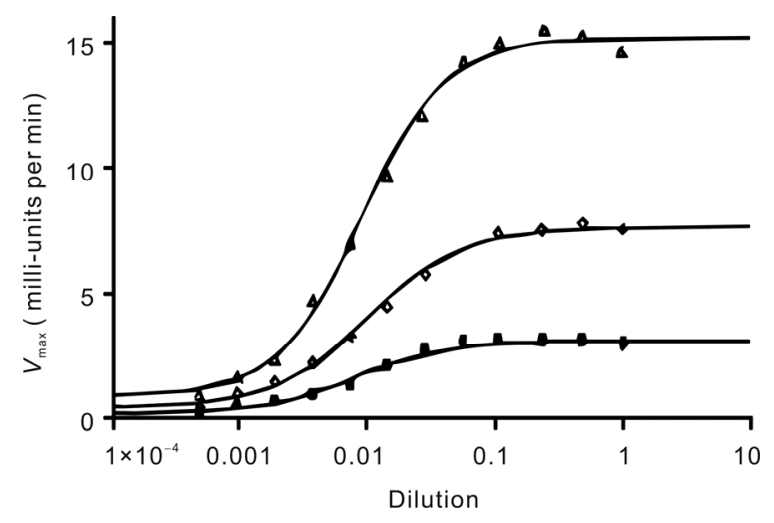

Figure 4 The effect of CNA $\Delta 316$ concentrations on $V_{\max }$. Each curve represents the relationship between $\mathrm{CNB}$ dilutions and maximum pNPP decomposition rate at the specified CNA $\triangle 316$ concentrations. CNA $\triangle 316$ concentrations, bottom to top, were: $2.5 \mu \mathrm{g} \mathrm{mL}^{-1}, 5 \mu \mathrm{g} \mathrm{mL}^{-1}$, and $10 \mu \mathrm{g} \mathrm{mL}^{-1}$. 
resent pre-dilution factors of CNB sample and CNB reference material, respectively; Es represents the dilution factor of a CNB sample that generates a response equivalent to that generated by $50 \%$ effective concentration of CNB reference material; Er represents the dilution of CNB reference material at $50 \%$ effective concentration. Accurate protein quantification of $\mathrm{CNB}$ samples may not be required for the referral of biological activity values when this formula is employed. The method does not require accurate protein quantification or biological activity determination of CNA $\triangle 316$ (Figure 4), simplifying the calculations and minimizing the error associated with computed referrals of biological activities. Our method for determination of CNB activity established herein is specific and controlled, laying a solid foundation for CNB quality control testing.

Information about the CNA $\Delta 316-\mathrm{CNB}$ complex was obtained from our data, as follows. (i) Results described in the "An assay for measuring the activity of CNB" section indicated that the biological activity of CNA $\triangle 316$ can be increased up to 18.6 -fold by CNB. (ii) Molar ratio of $\mathrm{CNA} \triangle 316$ to $\mathrm{CNB}$ in the CNA $\triangle 316-\mathrm{CNB}$ complex was approximated using the straight line section of three four-parameter curves in Figure 3. Molar equivalents of $\mathrm{CNB}$ and CNA $\triangle 316$ varied geometrically and in equal proportions, suggesting that the same amount of CNA $\triangle 316$ and $\mathrm{CNB}$ was required for the formation of the CNA $\triangle 316-\mathrm{CNB}$ complex. The molar ratio of $\mathrm{CNA} \triangle 316$ to $\mathrm{CNB}$ in the CNA $\triangle 316-C N B$ complex was therefore estimated as $1: 1$. (iii) Dissociation constant $\left(K_{\mathrm{d}}\right)$ of the CNA $\Delta 316-\mathrm{CNB}$ complex was also estimated. The formula $K_{\mathrm{d}}=([\mathrm{A}]-[\mathrm{AB}])$ $([\mathrm{B}]-[\mathrm{AB}]) /[\mathrm{AB}]$ can be applied depending on the nature of the compound (Marston et al., 1975; Maekawa, 1975; Kandutsch et al., 1981). Herein, [A] and [B] represent the initial molar concentration of CNA $\triangle 316$ and the concentration of free $\mathrm{CNB}$ in the assay, respectively, and $[\mathrm{AB}]$ represents the molar concentration of the complex formed in the assay. $([\mathrm{A}]-[\mathrm{AB}])$ and $([\mathrm{B}]-[\mathrm{AB}])$ represent molar concentration of $\mathrm{CNA} \triangle 316$ and the equilibrium concentration of $\mathrm{CNB}$, respectively. Any point on the four-parameter curve satisfies the above equation, and at 50\% effective dose (ED50), the molar concentrations $([\mathrm{A}]-[\mathrm{AB}])$ and $[\mathrm{AB}]$ are equal. The equation can then be simplified to $K_{\mathrm{d}}=([\mathrm{B}]-[\mathrm{A}]) / 2$. Furthermore, $K_{\mathrm{d}}$ can be estimated using the ratio of molar concentrations of the CNA $\triangle 316-C N B$ complex and $\mathrm{CNA} \Delta 316$. A point on the four-parameter curve reflected this ratio, and the calculated $K_{\mathrm{d}}$ was in the range of $2.83 \times 10^{-6}$ to $2.65 \times 10^{-7} \mathrm{~mol} \mathrm{~L}^{-1}$. This was close to the previously calculated CNA $K_{\mathrm{d}}$ range (Rusnak and Mertz, 2000).

To conclude, we established a method for the determination of biological activity of CNB. This method is simple and easily standardized, and lays foundations for drug development of CNB.

\section{MATERIALS AND METHODS}

\section{Proteins, materials, and reagents}

Lyophilized CNA $\Delta 316$, CNB standard, CNB test sample (Beijing Normal University), p-nitrophenylphosphate (pNPP) (34047, Thermo Fisher Science, USA), bovine serum albumin (BSA) (ST023 Beyotime, Shanghai), DLdithiothreitol (DTT) (ST040, Beyotime, Shanghai), and anti-CNB polyclonal antibody (ab154650, Abcam, USA) were used.

Testing medium comprised $50 \mathrm{mmol} \mathrm{L}{ }^{-1}$ Tris- $\mathrm{HCl}(\mathrm{pH}$ 7.4), $0.2 \mathrm{mg} \mathrm{mL}^{-1} \mathrm{BSA}, 20 \mathrm{mmol} \mathrm{L}{ }^{-1} \mathrm{pNPP}, 1 \mathrm{mmol} \mathrm{L}^{-1}$ DTT, $1 \mathrm{mmol} \mathrm{L}{ }^{-1} \mathrm{MnCl}_{2}$, and $1 \mathrm{mmol} \mathrm{L}^{-1} \mathrm{CaCl}_{2}$. Microtiter plate reader (Spectrum plus 5, Molecular Device, USA) was used in the assays.

\section{CNB bioassay}

To test the activity of CNB, CNB samples and standards were reconstituted in water and diluted to $100 \mu \mathrm{g} \mathrm{mL}^{-1}$ with testing medium. Then, 12 two-fold dilutions were serially prepared in a 96 -well microtiter plate $(100 \mu \mathrm{L}$ per well), in duplicate. CNA $\triangle 316$ was reconstituted in water and diluted to $2.5-10 \mu \mathrm{g} \mathrm{mL} \mathrm{m}^{-1}$ with testing medium. The solution was then added to the same $96-w e l l$ plate $(100 \mu \mathrm{L}$ per well). The above two steps were performed on ice.

The absorbance was read with a microtiter plate reader pre-warmed to $30^{\circ} \mathrm{C}$, at $410 \mathrm{~nm}$, in an enzyme kinetics mode. $\mathrm{A}_{410}$ values were read every $5 \mathrm{~min}$ for $3 \mathrm{~h}$. The results were recorded. The data were analyzed using a fourparameter regression method.

The maximum decomposing velocity $\left(V_{\max }\right)$ in each well was calculated using an enzyme kinetics curve. CNB dilution and $V_{\max }$ values were fitted to a four-parameter regression equation commonly used for curve-fitting analyses in bioassays. The following is the four-parameter regression model, with the independent value $X$ and dependent value $Y$ : $Y=(\mathrm{A}-\mathrm{D}) /\left(1+(X / \mathrm{C})^{\wedge} \mathrm{B}\right)+\mathrm{D} ; \mathrm{A}$, upper asymptote valuation. $\mathrm{D}$, lower asymptote valuation. $\mathrm{B}$, slope of the curve. $\mathrm{C}$, half the maximum binding dose. Here, we defined CNB dilution as $X$, and $V_{\max }$ as $Y$.

\section{Parallelism analysis}

Reaction consistency between the reference materials and the samples was assessed using the distances between the inflection points of the four-parameter regression equation curve. Data values that were parallel between the reference materials and the sample were analyzed using the regression coefficient $t$ test.

\section{Accuracy testing}

CNB reference dilutions, CNB test solutions, and mixed dilutions (dilutions of $\mathrm{CNB}$ references mixed with CNB test solutions at $1: 1$ ratio) were prepared. The test was per- 
formed in 96-well plates, as specified in the "CNB bioassay" section, and the recovery was calculated.

\section{Precision testing}

Independent assays were carried out on different days. Every day, the assays were performed in three different 96-well microtiter plates. Inter- and intra-plate precisions were calculated.

\section{Linearity, linear range, and sensitivity}

Linearity, linear range, and sensitivity were analyzed using the four-parameter regression equation.

\section{Specificity testing}

The specificity of the method was evaluated by determining the effect of therapeutic cytokines, including recombinant human granulocyte colony-stimulating factor (rhG-CSF), recombinant human Interferon- $\alpha 2 b$ (rhIFN- $\alpha 2 b$ ), and recombinant human Interleukin-2 (rhIL-2), on the OD values measured. Serial adjusted dilutions were prepared as described in method CNB bioassay.

Excess of CNB polyclonal antibody was used to neutralize $\mathrm{CNB}$, at $37^{\circ} \mathrm{C}$ for $30 \mathrm{~min}$, before activity assays.

\section{Robustness testing}

Different concentrations of CNA $\Delta 316$ were pipetted into wells of a 96-well microtiter plate to determine the effect of different CNA $\Delta 316$ concentrations on assay read-outs.

Compliance and ethics The author(s) declare that they have no conflict of interest.

Acknowledgements This work was supported by the National Important Novel Medicine Research Project (2012ZX09304010, 2013ZX09102062), and the National Natural Science Foundation of China (31270849).

Aramburu, J., Rao, A., and Klee, C.B. (2000). Calcineurin: from structure to function. Curr Top Cell Regul 36, 237-295.

Cheng, J., Tang, W., Su, Z., Guo, J., Tong, L., and Wei, Q. (2012). Calcineurin subunit B promotes TNF-alpha-induced apoptosis by binding to mitochondria and causing mitochondrial $\mathrm{Ca}^{2+}$ overload. Cancer Lett 321, 169-178.

Chaudhuri, G., Chatterjee, S., and Venu-Babu, P. (2013). Kinetic behav- iour of calf intestinal alkaline phosphatase with pNPP. Indian J Biochem Biophys 50, 64-71.

Feng, B., and Stermmer, P.M. (1999). Interactions of calcineurin A, calcineurin $\mathrm{B}$, and $\mathrm{Ca}^{2+}$. Biochemistry 38, 12481-12489.

Jin, F.Z., Lian, M.L., Wang, X., and Wei, Q. (2005). Studies of the anticancer effect of calcineurin B. Immuno pharmacol Immunotoxicol 27, 199-210.

Kissmehl, R., Treptau, T., and Kottwitz, B. (1997). Occurrence of a para-nitrophenyl phosphate-phosphatase with calcineurin-like characteristics in Paramecium tetraurelia. Arch Biochem Biophys 344, 260-270.

Kandutsch. A.A., and Shown, E.P. (1981). Assay of oxysterol-binding protein in a mouse fibroblast, cell-free system. Dissociation constant and other properties of the system. J Biol Chem 256, 13068-13073

Liu, L., Su, Z., Xin, S., Cheng, J., Li, J., and Xu, L. (2012). The calcineurin $\mathrm{B}$ subunit $(\mathrm{CnB})$ is a new ligand of integrin $\alpha \mathrm{M}$ that mediates CnB-induced Apo2L/TRAIL expression in macrophages. Immunol $188,238-247$.

Marston, S., and Weber, A. (1975). Dissociation-constant of actin-heavy meromyosin subfragment-1 complex. Biochemistry 14, 3868-3873.

Maekawa, S. (1978). Dissociation constant of Tetrahymena tubulin-colchicine complex. J Biol Chem 84, 641-646.

Maynes, J.T., Perreault, K.R., and Cherney, M.M. (2004). Crystal structure and mutagenesis of a protein phosphatase-1: calcineurin hybrid elucidate the role of the beta12-beta13 loop in inhibitor binding. J Biol Chem 279, 43198-43206.

National Pharmacopoeia Committee. (2015). Pharmacopoeia of People's Republic of China Vol III, general 3525.

Rusnak, F., and Mertz, P. (2000). Calcineurin: form and function. Physiol Rev 80, 1483-1521.

Reiter, T.A., Abraham, R.T., Choi, M., and Rusnak, F. (1999). Redox regulation of calcineurin in T-lymphocytes. J Biol Inorg Chem 4, 632-644.

Su, Z., Xin, S., Xu, L., Cheng, J., Guo, J., and Li, L. (2012). The calcineurinB subunit induces TNF-related apoptosis-inducing ligand (TRAIL) expression via CD11b-NF- $\kappa$ B pathway in RAW264.7 macrophages. Biochem Biophys Res Commun 417, 777-783.

Wei,Q., Lian, M.L., Jing, F.Z., Zhang, N., Yan, M.S., Chen, Y., and Gao, Q.S. (2002). Studies of calcineurin B subunit from genetic engineering for use in medicine. Drug Dev Res 56, 40-43.

Wei, Q., Yan, M.S., Gao, Q.S., Jiang, G.H., Lian, M.L., and Chen, Y. (2003). Pharmaceutical composition containing calcineurin B subunit. PRC Patent, ZL 981 17642.9. 2003-02-05

Wei, Q., Yan, M.S., Gao, Q.S., Jiang, G.H., Lian, M.L., and Chen, Y. (2006). Pharmaceutical composition containing calcineurin B subunit. US Patent, 7,026,118 B1. 2006-04-11

Wei, Q., and Lee, E.Y. (1997). Mutagenesis of the L7 loop connecting beta strands 12 and 13 of calcineurin: evidence for a structural role in activity changes. Biochemistry 36, 7418-7424.

Yang, S.A., and Klee, C.B. (2000). Low affinity $\mathrm{Ca}^{2+}$-binding sites of calcineurin B mediate conformational changes in calcineurin A. Biochemistry $39,16147-16154$.

Open Access This article is distributed under the terms of the Creative Commons Attribution License which permits any use, distribution, and reproduction in any medium, provided the original author(s) and source are credited. 